\title{
Evaluation of Glycaemic Control, Glucose Variability and Hypoglycaemia on Long-Term Continuous Subcutaneous Infusion vs. Multiple Daily Injections: Observational Study in Pregnancies With Pre-Existing Type 1 Diabetes
}

\author{
Aleksandra Jotic - Tanja Milicic - Katarina Lalic • Ljiljana Lukic • \\ Marija Macesic · Jelena Stanarcic Gajovic - Milica Stoiljkovic • \\ Miroslava Gojnic Dugalic • Veljko Jeremic • Nebojsa M. Lalic
}

Received: December 29, 2019 / Published online: February 15, 2020

(C) The Author(s) 2020

\section{ABSTRACT \\ Introduction: We evaluated the effectiveness of long-term continuous subcutaneous insulin infusion (CSII) compared with multiple daily insulin (MDI) injections for glycaemic control}

Enhanced Digital Features To view enhanced digital features for this article go to https://doi.org/10.6084/ m9.figshare.11762346.

Electronic supplementary material The online version of this article (https://doi.org/10.1007/s13300020-00780-7) contains supplementary material, which is available to authorized users.

A. Jotic $(\bowtie) \cdot$ T. Milicic · K. Lalic · L. Lukic .

M. Macesic · J. Stanarcic Gajovic · M. Stoiljkovic ·

N. M. Lalic

Clinic for Endocrinology, Diabetes and Metabolic Diseases, Clinical Center of Serbia, Dr. Subotića 13, 11000 Belgrade, Serbia

e-mail: aleksandra.z.jotic@gmail.com

A. Jotic · T. Milicic - K. Lalic · L. Lukic .

M. Macesic · M. Gojnic Dugalic · N. M. Lalic Faculty of Medicine, University of Belgrade, Dr.

Subotića 8, 11000 Belgrade, Serbia

M. Gojnic Dugalic

Clinic for Gynecology and Obstetrics, Clinical Center of Serbia, Visegradska 26, 11000 Belgrade, Serbia

\section{Jeremic}

Department for Operations Research and Statistics, Faculty of Organizational Sciences, University of Belgrade, Belgrade, Serbia and variability, hypoglycaemic episodes and maternal/neonatal outcomes in pregnant women with pre-existing type 1 diabetes (pT1D).

Methods: Our observational cohort study included 128 consecutive pregnant women with pT1D, who were treated from 1 January 2010 to 31 December 2017. Of 128 participants, 48 were on CSII and 80 were on MDI. Glycaemic control was determined by glycated haemoglobin (HbA1c) (captured in preconception and each trimester of pregnancy). Glucose variability (GV) was expressed as the coefficient of variation $(\mathrm{CV})$ [calculated from self-monitoring of blood glucose (SMBG) values], and hypoglycaemia was defined as glucose values < $3.9 \mathrm{mmol} / \mathrm{l}$. The data on maternal and neonatal outcomes were collected from obstetrical records.

Results: Duration of the treatment was $8.8 \pm 5.3$ years in the CSII and $12.6 \pm 8.0$ years in the MDI group. The CSII lowered HbA1c in preconception $\quad(7.1 \pm 0.1$ vs. $7.9 \pm 0.2 \%$, $p=0.03)$ and the first $(6.9 \pm 0.1$ vs. $7.7 \pm 0.2 \%$, $p=0.02)$, second $(6.6 \pm 0.1$ vs. $7.2 \pm 0.1 \%$, $p=0.003)$ and third $(6.5 \pm 0.1$ vs. $6.8 \pm 0.1 \%$, $p=0.02$ ) trimesters significantly better than MDI. Significantly lower CV was observed only for fasting glycaemia in the first trimester (17.1 vs $28.4 \%, p<0.001)$ in favour of CSII. Moreover, the CSII group had significantly lower mean hypoglycaemic episodes/week/patient 
only during the first trimester $(2.0 \pm 1.7$ vs $4.8 \pm 1.5, p<0.01)$. In early pregnancy, the majority of women on CSII had less hypoglycaemia than on MDI (0-3: 79.1 vs. $29.1 \%$; $4-6$ : 18.8 vs. $65.8 \% ; \geq 7: 2.1$ vs. $5.1 \%, p<0.01$, respectively). We found no difference in the incidence of adverse maternal/neonatal outcomes.

Conclusions: Treatment with CSII resulted in a favourable reduction of $\mathrm{HbA} 1 \mathrm{c}$ in the preconception period and each trimester in pregnancy. Moreover, long-term CSII treatment demonstrated more stable metabolic control with less $\mathrm{GV}$ of fasting glycaemia and fewer hypoglyacemic episodes only during early pregnancy.

Keywords: Continuous subcutaneous insulin infusion; Glucose variability; Pregnancy; Type 1 diabetes

\section{Key Summary Points}

Why carry out this study?

Earlier comparative studies showed that continuous subcutaneous insulin infusion (CSII) in pregnant women with preexisting type 1 diabetes (pT1D) lowered glycated haemoglobin (HbA1c) to a greater extent than multiple daily insulin injections (MDI) in the first trimester, but a limited number of studies included preconception $\mathrm{HbA1c}$ in analysis. At the same time, there are scarce data about glucose variability $(\mathrm{GV})$, a factor that may contribute to glycaemic control, as well as the occurrence of hypoglycaemic episodes, during pregnancy with pT1D on different insulin treatments.

Therefore, the aim of our study was to evaluate the effectiveness of long-term CSII compared with MDI injections for glycaemic control and variability, hypoglycaemic episodes and maternal/neonatal outcomes in pregnant women with pT1D.

\section{What was learned from the study?}

We demonstrated the advantages of longterm CSII on glucose control due to the lower level of HbA1c achieved already in preconception and maintained during whole pregnancy. Also, we showed diminished GV and less frequent hypoglycaemic events only in the first trimester on CSII, an aspect of glycaemic control insufficiently evaluated in previous investigations.

In conclusion, treatment with CSII has resulted in favourable reduction of $\mathrm{HbA1c}$ in the preconception period and each trimester in pregnancy. Moreover, longterm CSII treatment demonstrated more stable metabolic control with less GV of fasting glycaemia and fewer hypoglycaemic episodes only during early pregnancy.

\section{INTRODUCTION}

It has been previously shown that achieving and maintaining target glycaemic control in pregnant women with pre-existing type 1 diabetes (pT1D) can be extremely challenging in clinical settings [1]. However, obtaining tight glycaemic control is highly recommended from preconception until delivery $[1,2]$.

During the first trimester, improved insulin sensitivity increases the risk of hypoglycaemia. However, in late gestation, insulin sensitivity decreases, which requires correction of pronounced postprandial glycaemic spikes, i.e., frequent adjustment of insulin doses [3].

Earlier comparative studies showed that continuous subcutaneous insulin infusion (CSII) in pregnant women with pT1D lowered glycated haemoglobin (HbA1c) to a greater extent than multiple daily insulin injections (MDIs) in the first trimester [4]. Furthermore, recent data suggested superiority of CSII treatment in reducing HbA1c during the whole pregnancy [5]. The latest meta-analysis on the 
topic revealed better glycaemic control on CSII during the first trimester, with a less pronounced beneficial effect in the second and third trimesters [6].

Previous studies also reported that women with pT1D showed higher glucose variability, with a twofold higher risk of hyperglycaemic excursions during the day, than healthy pregnant women [7]. However, the well-known indicator-HbA1c level-does not reflect glucose variability during pregnancy $[8,9]$. In this way, glucose variability is a factor that may contribute to glycaemic control, which is suggested from the recently obtained data based on continuous glucose monitoring (CGM) reports in pregnancy in pT1D, but more evaluation is required with respect to different types of available insulin treatment in pregnancies complicated by pT1D [10]. In this context, it was reported that pregnant women with pT1D using CGM spent more time in target and less time hyperglycaemic, and they had less glycemic variability, than did pregnant control participants, with comparable severe hypoglycaemic episodes and time spent hypoglycaemic [11]. In addition, results from a recently published study obtained in non-pregnant T1D patients suggested stable and long-term improvement in glycaemic parameters with CGM, irrespective of type of insulin delivery [12].

Besides, numerous studies showed an association of increased risk of adverse pregnancy outcome with higher levels of HbA1c during conception $[13,14]$.

To our knowledge, studies comparing CSII and MDI in pregnancy have had conflicting results due to different study designs, populations followed, initiations of CSII therapy, etc. In this context, some of them have shown an increase in maternal weight, neonatal hypoglycaemia and birth weight with CSII therapy [15-17].

A limited number of studies included preconception HbA1c in the analysis, and a minority showed lower HbA1c levels in the CSII group [6]. At the same time, there are scarce data on the occurrence of hypoglycaemic episodes during pregnancy with pT1D on different insulin treatments. Still, the effect of long-term
CSII therapy in pregnant women with pT1D on parameters of metabolic control from preconception till delivery and adverse pregnancy events remains to be clarified.

Within this context, the aim of our study was to evaluate the effectiveness of long-term CSII compared with MDI injections for glycaemic control and variability, hypoglycaemic episodes and maternal/neonatal outcomes in pregnant women with pT1D.

\section{METHODS}

\section{Study Design and Settings, Ethics and Informed Consent}

The study was an observational prospective cohort study and included 128 consecutive singleton pregnant women with pT1D who were treated at the Department for metabolic disorders, intensive treatment and cell therapy in diabetes [Clinic for Endocrinology, Diabetes and Metabolic Diseases in Belgrade (public clinic, tertiary care level)] for diabetes from 1 January 2010 to 31 December 2017.

Pregnant women had been completely informed about the study before giving informed consent to participate. All procedures followed were part of the standard medical care and in accordance with the ethical standards of the responsible committee on human experimentation (institutional and national) and the Helsinki Declaration of 1964, as revised in 2013. Approval of the study by an ethics committee was considered unnecessary because all patient data were extracted from the department's database and were anonymous, assuring that complete data protection of the participants was provided.

\section{Inclusion and Exclusion Criteria}

In general, CSII has been suggested for all women of childbearing potential with pT1D on MDI in case of unsatisfactory metabolic control related to treatment targets. Current treatment of MDI or CSII had been initiated at least 1 year before conception in women with pT1D. 
For the present observational study, 80 pregnant women were treated with MDI (MDI group) using a rapid insulin analogue (aspart) before meals and a bedtime basal analogue (detemir), while 48 were treated with CSII (Medtronic Paradigm 722, Medtronic Paradigm Veo, insulin aspart) based on boluses given before meals, and the basal infusion rate was adjusted according to the glycaemic targets for the fasting and preprandial state. Both insulin treatments were fully reimbursed by the National Health Insurance Fund. Women who initiated CSII during actual pregnancy, women with gestational diabetes or type 2 diabetes, pregnancy obtained from assisted reproductive technology, or twin pregnancies as well as women referred from the general hospital or with incomplete medical records were excluded from the study.

\section{Measurements and Definitions of Measurements}

In both groups we recorded: age, duration of T1D, current treatment (MDI or CSII) and gestational weight gain (GWG). These data were taken from medical records. Body mass index (BMI) was calculated using the equation BMI = weight $(\mathrm{kg}) /$ height $(\mathrm{m})^{2}$.

HbA1c in preconception and each trimester was measured using electrophoresis. Values for fasting, postprandial glycaemia as well as hypoglycaemia during trimesters were taken from standard logbooks. Hypoglycaemia was defined by glycaemia $<3.9 \mathrm{mmol} / \mathrm{l}$. Based on the number of hypoglycaemia episodes per week per patient, we divided all women into tertiles: rare (0-3 hypoglycaemia), moderate (4-6 hypoglycaemia) and frequent ( $\geq 7$ hypoglycaemia) hypoglycaemia episodes per week per patient. Glucose variability was determined by the coefficient of variation $(\mathrm{CV}=\mathrm{SD} / \overline{\mathrm{x}})$ of SMBG at different points (fasting or postprandial) during trimesters.

We also measured the total daily insulin requirement and calculated the insulin sensitivity factor (ISF) (using Eq. 100/total daily dose) and carbohydrate ratio (CHR) (using Eq. 500/total daily dose) in each trimester.

\section{Pregnancy Outcomes}

Maternal and foetal outcomes, including gestational hypertension, preeclampsia [diagnosed by an obstetrician: blood pressure $\geq 140$ / $90 \mathrm{mmHg}$ with proteinuria $(\geq 0.5 \mathrm{~g})]$, preterm delivery (before 37 completed gestational weeks), emergency caesarean section, gestational week at delivery, Apgar score in the first minute, birth weight, newborn large for gestational age (LGA; $>90$ th percentile according to growth charts for the Serbian population), newborn small for gestational age (SGA) $(<10$ th percentile according to growth charts for the Serbian population), macrosomia (birth weight $>4000 \mathrm{~g}$ ), stillbirth, neonatal hypoglycaemia (glycaemia $<1.5 \mathrm{mmol} / \mathrm{l}$ or requiring intravenous glucose infusion), congenital malformation, admission to the neonatal intensive care unit (NICU) and neonatal hyperbilirubinaemia, were collected from the obstetrical medical records [16].

\section{Model of Care: Preconception and Pregnancy}

Planning pregnancy was defined as having preconception counselling. Standard preconception care for women with pT1D and childbearing potential was applied for all women irrespective of their treatment with MDI or CSII [1]. Pregnancy was confirmed by ultrasound examination and women had regular prenatal obstetrics visits according to the National Guideline for Pregnancy Care at the Clinic for Gynaecology and Obstetrics, Clinical Centre of Serbia [18].

HbA1c was recorded before conception and measured in each trimester. In addition, pregnant women were asked to do self-monitoring of blood glucose (SMBG) at least five times a week (preferably more frequently) during the whole pregnancy. These measurements were taken per day with a glucometer, when fasting, before and $2 \mathrm{~h}$ after meals, at bedtime and during the night for a total of at least nine measurements. According to the current recommendations, suggested fasting targets were $<$ $5.3 \mathrm{mmol} / \mathrm{l}$ and $<6.8 \mathrm{mmol} / \mathrm{l} 2 \mathrm{~h}$ after meals, 
respectively [2]. Women were also asked to record hypoglycaemia, which was defined by glycaemia $<3.9 \mathrm{mmol} / \mathrm{l}$. SMBGs were reviewed and treatment adjusted at least once a week during the first trimester and twice a month or more frequently, if necessary, until delivery.

We advised women about optimal timing for prandial insulin (15 min before meals in early pregnancy and 30-40 min before meals in late pregnancy) [19]. In addition, all pregnant women with pT1D were re-educated about carbohydrate counting, and we suggested recording the composition of each meal to better adjust the insulin dose. Sixty-seven per cent of women on CSII used the bolus calculator option during pregnancy.

All instructions listed above regarding monitoring of metabolic control are part of the standard procedures implemented in usual clinical practice in pregnant woman with pT1D at our department.

The blood pressure target was a systolic blood pressure $<130 \mathrm{mmHg}$ and a diastolic blood pressure $<80 \mathrm{mmHg}$, measured routinely using a sphygmomanometer at each visit.

\section{Statistical Analysis}

Data are presented as mean \pm SD values and were tested for normal distribution using the Kolmogorov-Smirnov test. The differences were tested for significance using Student's $t$ test, while the chi-square test and Mann-Whitney were used for parametric and non-parametric data, respectively. The Levine $\mathrm{F}$ test was used to access differences between CVs. A two-sided $p$ value of 0.05 was considered statistically significant. Data were analysed using Statistical Package for the Social Sciences (SPSS) software (Advanced Statistics, version 22.0, Chicago, IL USA).

\section{RESULTS}

\section{Baseline Characteristics}

Clinical characteristics and duration of previous treatment (MDI or CSII) of pregnant women with pT1D are shown in Table 1 . There was no significant difference with respect to age and duration of T1D between groups (Table 1). Furthermore, we did not find any significant difference regarding BMI and GWG between the groups.

\section{Results Pertaining to Glycaemic Control}

In preconception, women on CSII showed lower levels of HbA1c than the MDI group $(7.1 \pm 0.1$ vs. $7.9 \pm 0.2 \%, p=0.03$ ). Moreover, HbA1c was significantly lower during trimesters in the CSII group compared with MDI [first $(6.9 \pm 0.1$ vs. $7.7 \pm 0.2 \%, p=0.02)$, second $(6.6 \pm 0.1$ vs. $7.2 \pm 0.1 \%, p=0.003)$ and the third $(6.5 \pm 0.1$ vs. $6.8 \pm 0.1 \%, p=0.02$ ) trimester] (Fig. 1).

We did not find any significant difference between average fasting plasma glucose levels from SMBG during pregnancy when comparing these two intensified insulin regimens (Fig. 2). Simultaneously, the groups were compared according to average PPG during the whole pregnancy excluding late gestation. In the third trimester, pregnant women with pT1D treated with CSII had a lower average PPG level after breakfast than the MDI group $(6.8 \pm 1.0 \mathrm{mmol} /$ 1 vs. $7.7 \pm 1.3, p<0.001$ ) (Fig. 2 c).

When we analysed the percentage of pregnant women who achieved target PPG levels, there was no difference between the groups (Fig. 3), except in late pregnancy. In the third trimester after breakfast, a significantly higher percentage of pregnant women on CSII reached their target 2-h PPG level (57.4 vs. $18.4 \%$, $p<0.001$ )(Fig. 3b).

\section{Results Related to Glycaemic Variability}

Pregnant women on CSII treatment had lower $\mathrm{CV}$ of SMBG measured fasting in the first trimester (17.1 vs. $28.4 \%, p<0.001)$ and after breakfast in the third trimester compared with the MDI group (14.1 vs. $17 \%, p=0.012)$ (Table 2).

\section{Results for Hypoglycaemia}

Analysing the mean number of hypoglycaemia episodes per week per patient, the CSII group 
Table 1 Baseline characteristics of pregnant women with pre-existing type 1 diabetes: MDI vs. CSII group

\begin{tabular}{llll}
\hline & MDI group $(\boldsymbol{n}=\mathbf{8 0})$ & CSII group $(\boldsymbol{n}=\mathbf{4 8})$ & $\boldsymbol{P}$ value \\
\hline Age (years) & $29.6 \pm 5.0$ & $27.6 \pm 5.6$ & 0.49 \\
Diabetes duration (years) & $13.1 \pm 8.2$ & $11.3 \pm 7.9$ & 0.98 \\
Duration of treatment (years) & $12.6 \pm 8.0$ & $8.8 \pm 5.3$ & NA \\
BMI $\left(\mathrm{kg} / \mathrm{m}^{2}\right)$ & $22.7 \pm 0.4$ & $22.9 \pm 0.4$ & 0.94 \\
GWG $(\mathrm{kg})$ & $11.5 \pm 0.5$ & $11.0 \pm 0.6$ & 0.66 \\
\hline
\end{tabular}

Data are mean $\pm S D$

$B M I$ body mass index, $G W G$ gestational weight gain

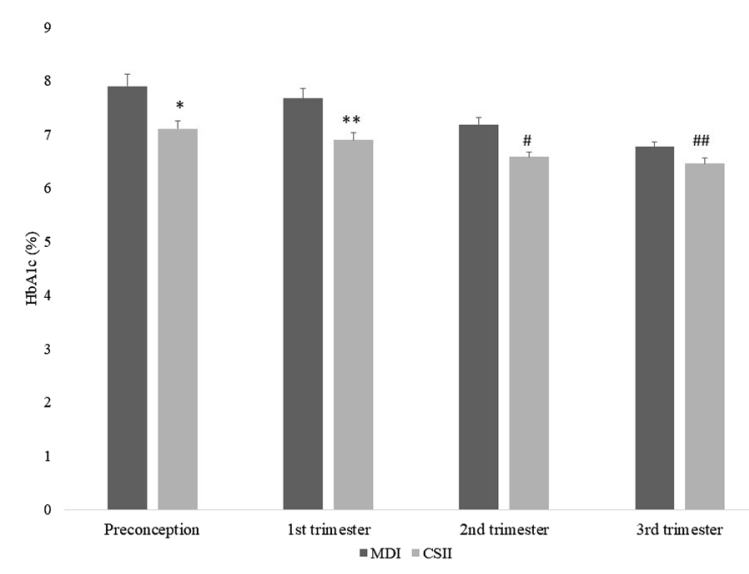

Fig. $1 \mathrm{HbAlc}$ in preconception and during trimesters in pregnant women with pT1D: comparison of different intensified insulin regimens: MDI (dark gray) vs. CSII (light gray). Values are mean \pm SE. Mann-Whitney test was used to evaluate the differences between groups. There was a statistically significant difference with respect to $\mathrm{HbAlc}$ in preconception and during pregnancy between groups. Pregnant women with pT1D on CSII had lower HbAlc levels in preconception $\left({ }^{*} p=0.03\right)$, the 1 st trimester $\left({ }^{* *} p=0.002\right)$ and 2 nd trimester $\left({ }^{*} p=0.003\right)$ as well as the 3 rd trimester $\left({ }^{\# \#} p=0.02\right)$ compared with MDI. Pre-existing type 1 diabetes, pT1D

had fewer hypoglycaemia episodes per week in the first trimester compared with MDI $(2.0 \pm 1.7$ vs. $4.8 \pm 1.5, p<0.01)$, but the difference diminished in the second and third trimester between groups (Fig. 4). At the same time, a higher percentage of women on CSII was in the first tertile (rare) compared with MDI in the first trimester (Fig. 5a) (0-3: 79.1 vs. $29.1 \%$; 4-6: 18.8 vs. $65.8 \%$; $\geq 7: 2.1$ vs. $5.1 \%, p<0.01$, respectively), while there was no difference in the second and third trimester (Fig. 5b, c).

\section{Results Related to Pregnancy Outcomes}

We found no difference in the incidence of gestational hypertension between groups, whereas the incidence of preeclampsia and SGA newborn could not be analysed because of a limited number of events. Groups were also comparable in the incidence of preterm delivery, emergency caesarean section, macrosomia, neonatal hypoglycaemia, admission to NICU as well as gestational week at delivery, Apgar score in the first minute and LGA newborns. Simultaneously, women in both groups delivered newborns with comparable neonatal weight, while newborns with neonatal hyperbilirubinaemia were found more often in the MDI group. We did not report congenital malformations in either groups (Table 3 ).

Table S1 represents the data regarding insulin requirements in both regimen groups, CSII and MDI, as well as the calculated CHR and ISF in each pregnancy trimester. Total insulin daily dose was significantly lower in the CSII compared with the MDI group in the first $(34.0 \pm 6.4$ vs. $41.6 \pm 10.3 \mathrm{U}, p=0.001)$, second $(38.5 \pm 8.7$ vs. $46.3 \pm 12.4 \mathrm{U}, p=0.001)$ and third trimester $(42.0 \pm 10.6$ vs. $52.0 \pm 16.8$ $\mathrm{U}, p=0.001)$. 

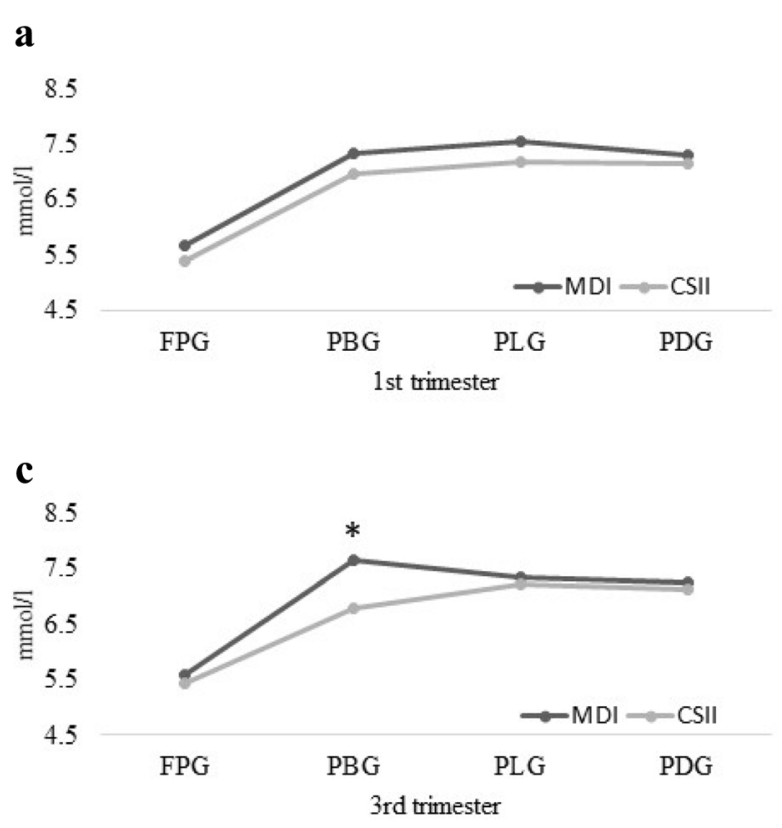

Fig. 2 Average fasting and postprandial glycaemia obtained from SMBG in pregnant women with pT1D: MDI vs. CSII. Data are presented as mean \pm SD values. $T$ test was used to evaluate the differences between groups. There was a statistically significant difference in the 3rd trimester in the CSII group due to the lower average PPG

\section{DISCUSSION}

In our observational study, which encompasses pregnant women with pT1D, we detected better glycaemic control on CSII than on MDI treatment, based on data evaluation from preconception through the trimesters till delivery. Moreover, the majority of pregnant women with pT1D achieved target glycaemic goals already in preconception and maintained them during the trimesters, using long-term CSII.

Data from previous studies focusing on glycaemic control in pregnant women with pT1D using different intensified insulin treatments (CSII or MDI) still remained inconsistent for superiority of either of them [4-6], partly because they did not focus on preconception monitoring or sustainability during the whole pregnancy. Moreover, glycaemic fluctuations, GV and hypoglycaemia, were suggested to influence the course of pregnancy in women with long-standing pT1D, but the effectiveness of different insulin treatments for glycaemic

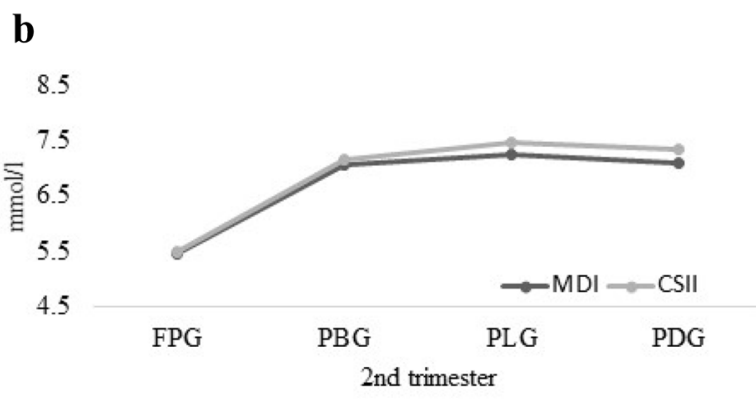

level after breakfast comparing with $\mathrm{MDI}\left(\mathrm{C} ;{ }^{*} p<0.001\right)$. $F P G$ fasting plasma glucose, $P B G$ postprandial breakfast glucose, $P L G$ postprandial lunch glucose, $P D G$ postprandial dinner glucose

control and variability and hypoglycaemic episodes in pregnant women with pT1D has not been elucidated [8].

Some prior studies in this field revealed better glycaemic control with CSII treatment in complicated T1D during pregnancy [20], in line with our study results showing lower HbA1c levels among women with long-standing T1D and CSII.

A recently published meta-analysis comprising 47 predominantly non-interventional studies showed higher HbA1c reduction in early pregnancy, but this advantage was diminished in the second and third trimesters [6].

However, some previous investigations suggested a comparable effect of CSII and MDI on glycaemic control [21]. Nevertheless, most of these available studies included pregnant women in the second trimester or the ones initiating pump treatment during actual pregnancy.

Moreover, a limited number of studies reported better preconception glycaemic 

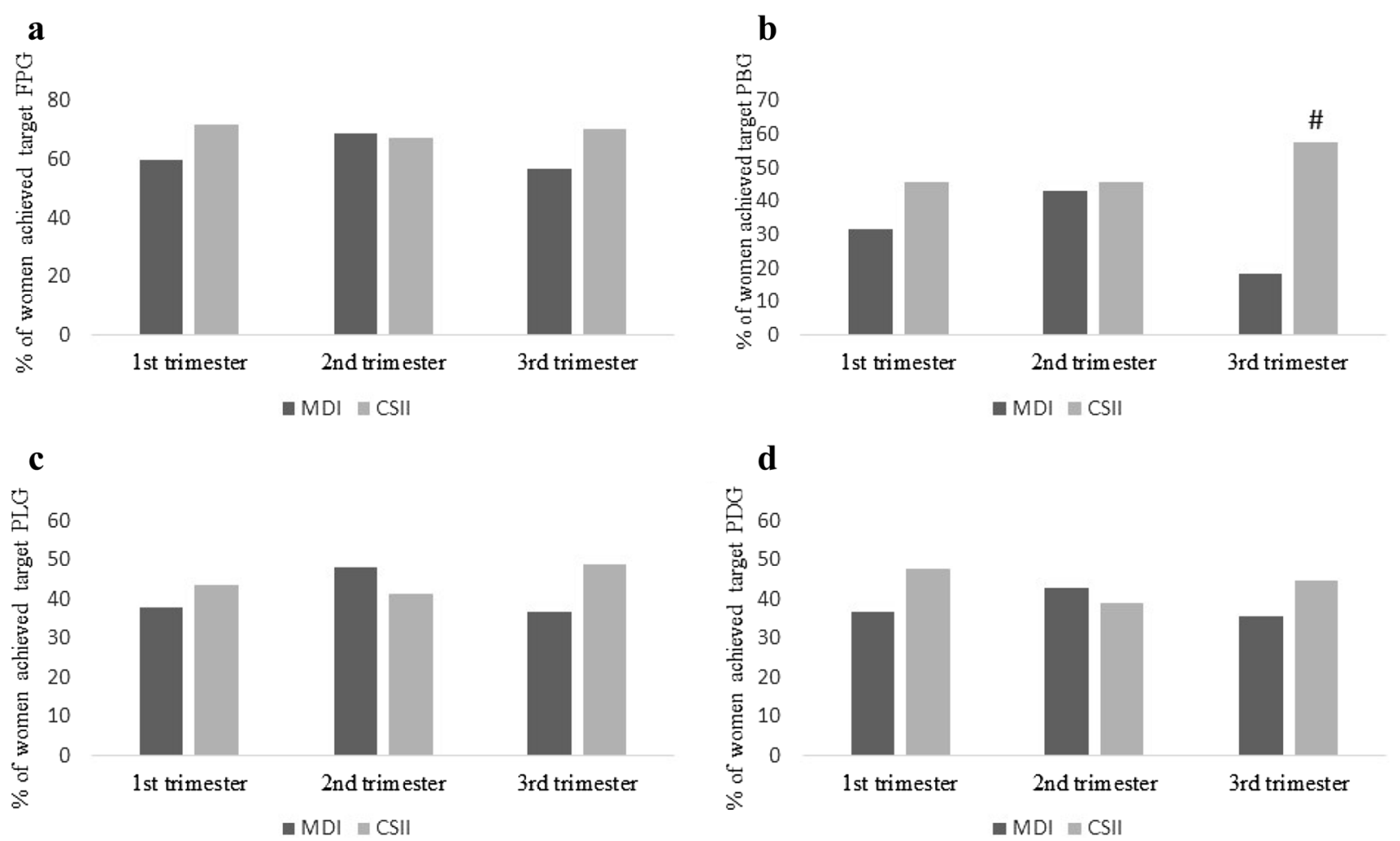

Fig. 3 Percentage of pregnant women with pT1D achieved target FPG (a) and PPG (b-d): MDI vs. CSII. Data are presented as \%. Chi-square test was used to evaluate the differences between groups. There was a statistically significant difference in the 3 rd trimester

control in women with pT1D on CSII compared with MDI treatment [22]. Our results also confirmed a lower HbA1c level in preconception in women on CSII than in those on MDI treatment.

Although our women were not randomized to the MDI or CSII treatment arm, we tried to overcome potential bias by homogenizing groups in respect to age, duration of disease and treatment. In our study, in contrast to other studies on the topic, we followed pregnant women with long-standing T1D and a longterm use of either type of insulin treatment before pregnancy.

During pregnancy, we focused not only on HbA1c, having in mind it might not adequately reflect different aspects of glucose control, but also analysed acute glucose fluctuations or GV, which contribute to adverse pregnancy events [8]. In addition, a better understanding of the pattern of blood glucose fluctuations in all three

among CSII group because of the higher percentage of women reaching their target $2 \mathrm{~h}$ after breakfast compared with MDI $(\mathbf{b} ; \stackrel{\#}{p} p<0.001)$

trimesters of pregnancy could make it easier to optimize glycaemic control in pregnant women with diabetes [7]. In that context, in our study we used $\mathrm{CV}$ as an established parameter of GV. Pregnant women with pT1D on CSII treatment had a lower CV of fasting glycaemia in early pregnancy as well as after breakfast in late pregnancy (obtained from SMBG). In that context, our results might imply that assessing $\mathrm{CV}$ from SMBG, even without the use of a more sophisticated and recently established standard method of blood glucose measurement, might be useful and clinically relevant as a surrogate marker of GV. Furthermore, in a recently published meta-analysis a significant number of studies, nearly half of those included, used only SMBG as a glucose monitoring method [6].

In addition, our findings are in line with previously published studies showing no difference in mean FPG and PPG between CSII and MDI in the first and second trimesters [23-25]. 
Table 2 CV of FPG and PPG obtained from SMBG among pregnant women with pT1D: MDI vs. CSII

\begin{tabular}{|c|c|c|c|c|}
\hline & $\begin{array}{l}\text { CV of } \\
\text { SMBG (\%) }\end{array}$ & $\begin{array}{l}\text { MDI } \\
(n=80)\end{array}$ & $\begin{array}{l}\text { CSII } \\
(n=48)\end{array}$ & $P$ value \\
\hline \multirow[t]{4}{*}{$\begin{array}{l}1 \text { st } \\
\text { trimester }\end{array}$} & FPG & 28.4 & 17.1 & $<0.001$ \\
\hline & PBG & 24.2 & 20.4 & 0.22 \\
\hline & PLG & 23.4 & 19.9 & 0.24 \\
\hline & PDG & 14.1 & 13.8 & 0.85 \\
\hline \multirow[t]{4}{*}{$\begin{array}{l}\text { 2nd } \\
\text { trimester }\end{array}$} & FPG & 23.9 & 23.2 & 0.81 \\
\hline & PBG & 24.1 & 23.1 & 0.75 \\
\hline & PLG & 23.5 & 21.4 & 0.5 \\
\hline & PDG & 14.8 & 13.7 & 0.56 \\
\hline \multirow[t]{4}{*}{$\begin{array}{l}\text { 3rd } \\
\quad \text { trimester }\end{array}$} & FPG & 21.3 & 23.8 & 0.4 \\
\hline & PBG & 17.0 & 14.1 & 0.01 \\
\hline & PLG & 18.7 & 23.7 & 0.08 \\
\hline & PDG & 12.1 & 14.6 & 0.16 \\
\hline
\end{tabular}

Data are present as CV (\%)

Coefficient of variation was calculated using the equation $\mathrm{SD} / \bar{x}$ of SMBG in different points (fasting or postprandial)

Levine $F$ test was used to access the difference between CVs

Significance was found in the CSII group concerning FPG in the first and PBG in the third trimester measuring the CV of SMBG

Comparisons in boldface type indicate significant differences $(p<0.05)$

$F P G$ fasting plasma glucose, $P B G$ postprandial breakfast glucose, $P L G$ postprandial lunch glucose, $P D G$ postprandial dinner glucose
However, according to our results, pregnant women with pT1D using CSII had lower mean PPG levels (after breakfast) in late pregnancy, which is an advantage in clinical settings. Moreover, besides better PPG regulation, a higher percentage of pregnant women with pT1D on CSII in our study reached the target value for the PPG goal in the third trimester after breakfast.

On the other hand, data on hypoglycaemic episodes in pregnant women with pT1D are quite limited. It has been shown that compared with MDI, CSII was associated with a slightly lower HbA1c level and a smaller risk of severe hypoglycaemia [26]. Also, it is important to note that the effort to achieve better glycaemic control together with less GV could predispose to hypoglycaemia. Our results are in agreement with studies showing the superiority of CSII regarding fewer hypoglycaemic episodes during early pregnancy $[1,27]$, which is an important clinical challenge because pregnant women with pT1D are most vulnerable to hypoglycaemia in that period [28]. As we have shown in this study, in respect to number of hypoglycaemia episodes per week, the majority of women on CSII were in the first tertile (rare hypoglycaemic events), which implies more stable glycaemic control with less GV and hypoglycaemia. In addition, the CSII group had lower TDD compared with MDI, in line with previously published data [6].

Current data on the relationship between glucose variability and adverse foetal outcome are still conflicting. Recent studies using CGM reported a correlation between parameters of glucose variability and foetal growth [29-31] in pregnant women with pT1D or gestational diabetes. Although earlier studies had conflicting results [32], in our study we demonstrated an availability of decreased glucose variability on CSII, even in clinical settings without the use of CGM, which might be of significant clinical relevance.

Concerning pregnancy outcomes, we could not demonstrate that CSII treatment induced a lower incidence of adverse pregnancy events compared with MDI therapy. The studies in this field gave conflicting results and, despite some positive results, most of the studies reported 


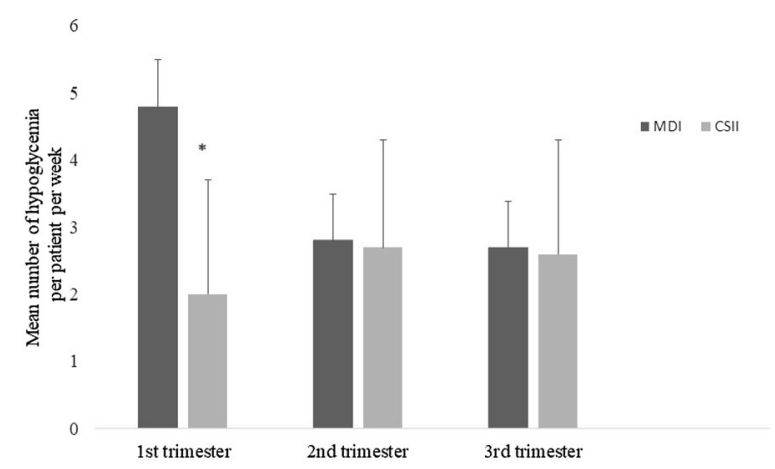

Fig. 4 Mean number of hypoglycaemic episodes per week per patient through trimesters in pregnant women with pT1D: MDI vs. CSII. Data are presented as mean \pm SD values. Mann-Whitney test was used to evaluate the differences between groups. There was a statistically significant difference in the 1st trimester in the CSII group due to a smaller mean number of hypoglycaemia episodes per week per patient than on MDI $\left({ }^{*} p<0.001\right)$

\section{a}

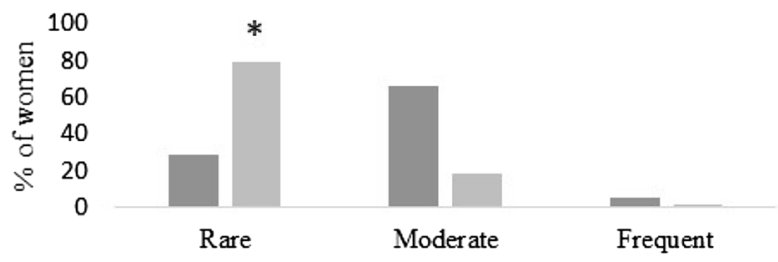

Number of hypoglycemic episodes per week during 1 st trimester

$$
\begin{aligned}
& \text { c }
\end{aligned}
$$

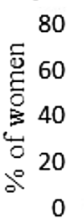$$
\text { MDI } \mathrm{CSII}
$$

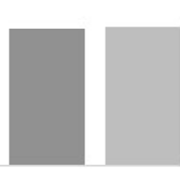

Rare

Number of hypoglycemic episodes per week in 3rd trimester

MDI $\mathrm{MCSII}$

Fig. 5 Percentage of pregnant women with pT1D distributed in tertiles according to number of hypoglycaemia episodes per week: MDI vs. CSII. Data are presented as \% of women. Mann-Whitney test was used to evaluate the differences between groups. There was a statistically significant difference in the first trimester in the CSII that optimal metabolic control during pregnancy does not always guarantee a favourable outcome [32, 33].

In addition, limitations of our study are the observational study design and use of a surrogate marker for glycaemic variability $\mathrm{CV}$ obtained by SMBG. Also, the relatively few adverse events are a limitation related to adequate evaluation of the incidence of adverse outcomes and do not allow formulating further conclusions.

Nonetheless, we demonstrated the advantages of long-term CSII on glucose control due to the lower level of HbA1c achieved already in preconception and maintained during the entire pregnancy. Also, we showed a diminished GV and less frequent hypoglycaemic episodes only in the first trimester on CSII, an aspect of glycaemic control insufficiently evaluated in previous investigations.

\section{b}

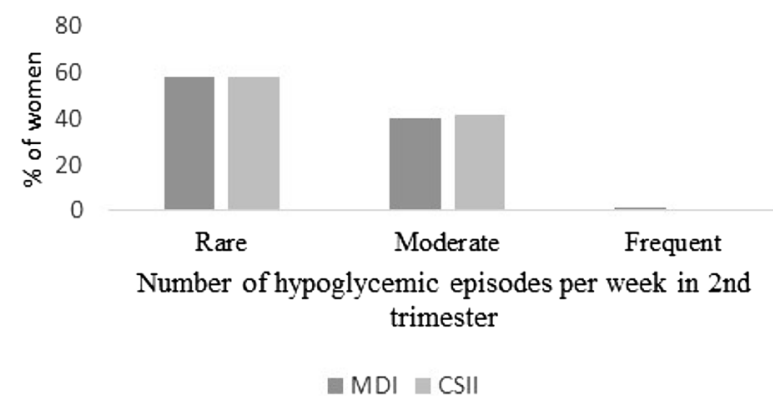

group due to the higher percentage of patients in the first tertile (rare) $(p<0.01)$. There were defined tertiles in relation to the number of hypoglycaemia episodes per week: rare (0-3 hypoglycaemia), moderate (4-6 hypoglycaemia) and frequent ( $\geq 7$ hypoglycaemia) 
Table 3 Adverse pregnancy events in pregnant women with pT1D: MDI vs. CSII

\begin{tabular}{llll}
\hline & MDI $(\boldsymbol{n}=\mathbf{8 0})$ & CSII $(\boldsymbol{n}=\mathbf{4 8})$ & $\boldsymbol{P}$ value \\
\hline Gestational week at delivery & $37.5 \pm 1.5$ & $37.1 \pm 1.4$ & 0.15 \\
Apgar score & $8.1 \pm 0.9$ & $8.07 \pm 1.5$ & 0.58 \\
Hypertension $(\%, n)$ & $25(20)$ & $12.8(6)$ & 0.09 \\
Preeclampsia $(\%, n)$ & $3.8(3)$ & $2.1(1)$ & NA \\
Preterm delivery $(\%, n)$ & $17.5(14)$ & $27.7(13)$ & 0.17 \\
Emergency caesarean section $(\%, n)$ & $16.3(13)$ & $25.5(12)$ & 0.2 \\
Macrosomia (\%, $n)$ & $19.2(15)$ & $14(6)$ & 0.46 \\
Stillbirth $(\%, n)$ & - & $2.1(1)$ & NA \\
Neonanatal hypoglycaemia $(\%, n)$ & $7.5(6)$ & $10.6(5)$ & 0.54 \\
NICU admission (\%, $n)$ & $10.7(8)$ & $17.4(8)$ & 0.28 \\
Neonatal hyperbilirubinaemia $(\%, n)$ & $30(24)$ & $14.9(7)$ & $\mathbf{0 . 0 0 5}$ \\
Neonatal weight (g) & $3456.7 \pm 801.4$ & $3459 \pm 639.4$ & 0.98 \\
LGA $(\%, n)$ & $50(40)$ & $44(21)$ & 0.59 \\
SGA (\%, $n$ ) & $4(5)$ & - & NA \\
Congenital malformation $(\%)$ & - & - & NA \\
\hline
\end{tabular}

Data are counts (percentages) or mean \pm SD

Significance was found for neonatal hyperbilirubinaemia in the CSII group

Comparisons in boldface type indicate significant differences $(p<0.05)$

Because of the limited number of events, significance could not be assessed in terms of preeclampsia, stillbirth and SGA $N I C U$ neonatal intensive care unit, $L G A$ large for gestational age, $S G A$ small for gestational age

\section{CONCLUSIONS}

In conclusion, treatment with CSII resulted in a favourable reduction of HbA1c in the preconception period and each trimester in pregnancy. Moreover, long-term CSII treatment demonstrated more stable metabolic control with less GV of fasting glycaemia and fewer hypoglycemic episodes only during early pregnancy.

\section{ACKNOWLEDGMENTS}

Funding. The study was funded by Project 175097 from the Ministry of Education, Science and Technological Development, Republic of
Serbia. No Rapid Service Fee was received by the journal for the publication of this article.

Authorship. All named authors meet the International Committee of Medical Journal Editors (ICMJE) criteria for authorship for this article, take responsibility for the integrity of the work as a whole, and have given their approval for this version to be published.

Author Contributions. Study concept and design: A. Jotic, T. Milicic, N. M. Lalić. Acquisition of data: A. Jotic, T. Milicic, K. Lalic, L. Lukic, M. Macesic, J. Stanarcic Gajovic, M. Stoiljkovic, M. Gojnic Dugalic. Analysis and interpretation of data: A. Jotic, T. Milicic, J. Stanarcic Gajovic, M. Stoiljkovic, N. M. Lalić. Drafting of the manuscript: A. Jotic, T. Milicic, J. Stanarcic Gajovic, M. Stoiljkovic, N. M. Lalić. 
Critical revision of the manuscript for important intellectual content: A. Jotic, T. Milicic, K. Lalic, J. Stanarcic Gajovic, M. Stoiljkovic, N. M. Lalić. Statistical analysis: V. Jeremic, A. Jotic, T. Milicic, M. Stoiljkovic. Obtained funding: N. M. Lalic. Administrative, technical, and material support: V. Jeremic, A. Jotic, T. Milicic, M. Stoiljkovic, N. M. Lalic. Study supervision: A. Jotic, T. Milicic, N. M. Lalić.

Disclosures. Aleksandra Jotic, Tanja Milicic, Katarina Lalic, Ljiljana Lukic, Marija Macesic, Jelena Stanarcic Gajovic, Milica Stoiljkovic, Miroslava Gojnic Dugalic, Veljko Jeremic and Nebojsa M. Lalic have nothing to disclose.

Compliance with Ethics Guidelines. Pregnant women were completely informed about the study before they gave informed consent to participate. All procedures followed were part of the standard medical care and in accordance with the ethical standards of the responsible committee on human experimentation (institutional and national) and with the Helsinki Declaration of 1964, as revised in 2013. Approval of the study by an ethics committee was considered unnecessary because all patient data were extracted from the Department's database and were anonymous, assuring that complete data protection of the participants was provided.

Data Availability. Access to the database is currently strictly regulated by law and therefore the data will not be deposited.

Open Access. This article is licensed under a Creative Commons Attribution-NonCommercial 4.0 International License, which permits any non-commercial use, sharing, adaptation, distribution and reproduction in any medium or format, as long as you give appropriate credit to the original author(s) and the source, provide a link to the Creative Commons licence, and indicate if changes were made. The images or other third party material in this article are included in the article's Creative Commons licence, unless indicated otherwise in a credit line to the material. If material is not included in the article's Creative Commons licence and your intended use is not permitted by statutory regulation or exceeds the permitted use, you will need to obtain permission directly from the copyright holder. To view a copy of this licence, visit http://creativecommons.org/licenses/by$\mathrm{nc} / 4.0 /$.

\section{REFERENCES}

1. American Diabetes Association. Management of diabetes in pregnancy: standards of medical care in diabetes-2019. Diabetes Care. 2019;42(Supplement 1):S165-72.

2. Jensen DM, Korsholm L, Ovesen P, Beck-Nielsen H, Moelsted Pedersen L, Westergaard JG, et al. Periconceptional $\mathrm{A} 1 \mathrm{C}$ and risk of serious adverse pregnancy outcome in 933 women with type 1 diabetes. Diabetes Care. 2009;32(6):1046-8.

3. Lenhard MJ, Kinsley BT. Insulin therapy for the treatment of type 1 diabetes during pregnancy. J Matern Fetal Neonatal Med. 2014;12:1270-5.

4. Lapolla A, Dalfrà MG, Masin M, Bruttomesso D, Piva I, Crepaldi C, Tortul C, Dalla Barba B, Fedele D. Analysis of outcome of pregnancy in type 1 diabetics treated with insulin pump or conventional insulin therapy. Acta Diabetol. 2003;40:143-9.

5. Kallas-Koeman MM, Kong JM, Klinke JA, Butalia S, Lodha AK, Lim KI, Duan QM, Donovan LE. Insulin pump use in pregnancy is associated with lower HbA1c without increasing the rate of severe hypoglycaemia or diabetic ketoacidosis in women with type 1 diabetes. Diabetologia. 2014;57(4):681-9.

6. Rys PM, Ludwig-Slomczynska AH, Cyganek K, Maleck MT. Continuous subcutaneous insulin infusion vs multiple daily injections in pregnant women with type 1 diabetes mellitus: a systematic review and meta-analysis of randomised controlled trials and observational studies. Eur J Endocrinol. 2018;178:545-63.

7. Dalfrà MG, Chilelli NC, Di Cianni G, Mello G, Lencioni C, Biagioni S, Scalese M, Sartore G, Lapolla A. Glucose fluctuations during gestation: an additional tool for monitoring pregnancy complicated by diabetes. Int $\mathrm{J}$ Endocrinol. 2013. Article ID 279021, 8 pages.

8. Dalfra MG, Sartore G, Cianni GD, et al. Glucose variability in diabetic pregnancy. Diabetes Technol Ther. 2011;13(8):853-9. 
9. Hawkins JS. Glucose monitoring during pregnancy. Curr DiabRep. 2010;10:229-34.

10. Battelino T, Danne T, Bergenstal RM, et al. Clinical targets for continuous glucose monitoring data interpretation: recommendations from the international consensus on time in range. Diabetes Care. 2019;42:1593-603.

11. Feig DF, Donovan LE, Corcoy R, Murphy KE, Amiel $\mathrm{SA}$, Hunt KF, et al. Continuous glucose monitoring in pregnant women with type 1 diabetes (CONCEPTT): a multicentre international randomised controlled trial. Lancet. 2017;390:2347-59.

12. Soupal J, Petruzelkova L, Grunberger G, et al. Glycemic outcomes in adults with T1D are impacted more by continuous glucose monitoring than by insulin delivery method: 3 years of follow-up from the COMISAIR study. Diabetes Care. 2019. https:// doi.org/10.2337/dc19-0888. [Epub ahead of print].

13. Suhonen L, Hiilesmaa V, Teramo K. Glycaemic control during early pregnancy and fetal malformations in women with type I diabetes mellitus. Diabetologia. 2000;43:79-82.

14. Feldman AZ, Brown FM. Management of type 1 diabetes in pregnancy. Curr Diab Rep. 2016;16:76.

15. Cyganek K, Hebda-Szydlo A, Katra B, et al. Glycemic control and selected pregnancy outcomes in type 1 diabetes women on continuous subcutaneous insulin infusion and multiple daily injections: the significance of pregnancy planning. Diabetes Technol Ther. 2010;12:41-7.

16. Chen R, Ben-Haroush A, Weismann-Brenner A, et al. Level of glycemic control and pregnancy outcome in type 1 diabetes: a comparison between multiple daily insulin injections and continuous subcutaneous insulin infusions. Am J Obstet Gynecol. 2007; 197:404.e1-e5.

17. Farrar D, Tuffnell DJ, West J: Continuous subcutaneous insulin infusion versus multiple daily injections of insulin for pregnant women with diabetes. Cochrane Database Syst Rev 2007:CD005542.

18. Nacionalni vodič za lekare $\mathrm{u}$ primarnoj zdravstvenoj zaštiti. Zdravstvena zaštita žena u toku trudnoće.Republička stručna komisija za izradu i implementaciju vodiča u kliničkoj praksi, Ministarstvo zdravlja Republike Srbije, Srpsko lekarsko društvo, 2005.

19. Luijf YM, van Bon AC, Hoekstra JB, Devries JH. Premeal injection of rapid-acting insulin reduces postprandial glycemic excursions in type 1 diabetes. Diabetes Care. 2010;33:2152-5.
20. Kekalainen P, Juuti M, Walle T, Laatikainen $\mathrm{T}$. Continuous subcutaneous insulin infusion during pregnancy in women with complicated type 1 diabetes is associated with better glycemic control but not with improvement in pregnancy outcomes. Diabetes Technol Ther. 2016;18(3):144-50.

21. Bruttomesso D, Bonomo M, Costa S, et al. Type 1 diabetes control and pregnancy outcomes in women treated with continuous subcutaneous insulin infusion (CSII) or with insulin glargine and multiple daily injections of rapid-acting insulin analogues (glargine-MDI). Diabetes Metab. 2011;37(5):426-31.

22. Neff KJ, Forde R, Gavin C, et al. Pre-pregnancy care and pregnancy outcomes in type 1 diabetes mellitus: a comparison of continuous subcutaneous insulin infusion and multiple daily injection therapy. Ir J Med Sci. 2014;183:397-403.

23. Mello G, Biagioni S, Ottanelli S. Continuous subcutaneous insulin infusion (CSII) versus multiple daily injections (MDI) of rapid acting insulin analogues and detemir in type 1 diabetic (T1D) pregnant women. J Matern Fetal Neonatal Med. $2015 ; 28(3): 276-80$.

24. Murphy HR, Elleri D, Allen JM, et al. Pathophysiology of postprandial hyperglycaemia in women with type 1 diabetes during pregnancy. Diabetologia. 2012;55:282-90.

25. Abell SK, Suen M, Pease A, et al. Pregnancy outcomes and insulin requirements in women with type 1 diabetes treated with continuous subcutaneous insulin infusion and multiple daily injections: cohort study. Diabetes Technol Ther. 2017;19(5):280-7.

26. Pickup JC, Sutton AJ. Severe hypoglycaemia and glycaemic control in type 1 diabetes: meta-analysis of multiple daily insulin injections compared with continuous subcutaneous insulin infusion. Diabet Med. 2008;25:765-74.

27. Wender-Ozegowska E, Zawiejska A, Ozegowska K, et al. Multiple daily injections of insulin versus continuous subcutaneous insulin infusion for pregnant women with type 1 diabetes. Aust N Z J Obstet Gynaecol. 2013;53:130-5.

28. Ringholm L, Pedersen-Bjergaard U, Thorsteinsson B, Damm P, Mathiesen ER. Hypoglycaemia during pregnancy in women with Type 1 diabetes: hypoglycaemia during pregnancy in women with type 1 diabetes. Diabet Med. 2012;29:558-66.

29. McGrath RT, Glastras SJ, Seeho SK, Scott ES, Fulcher GR, Hocking SL. Association between glycemic variability, $\mathrm{HbA}_{1 \mathrm{c}}$, and large-for-gestational-age 
neonates in women with type 1 diabetes. Diabetes Care. 2017;40(8):e98-100.

30. Law GR, Ellison GT, Secher AL, et al. Analysis of continuous glucose monitoring in pregnant women with diabetes: distinct temporal patterns of glucose associated with large-for-gestationalage infants. Diabetes Care. 2015;38:1319-25.

31. Gupta R, Khoury J, Altaye M, Dolan L, Szczesniak RD. Glycemic excursions in type 1 diabetes in pregnancy: a semiparametric statistical approach to identify sensitive time points during estation. J Diabetes Res. 2017;2017:2852913.

32. Polsky S, Garcetti R. CGM, pregnancy, and remote monitoring. Diabetes Technol Ther. 2017;19(S3): S49-59.

33. Yu Q, Aris IM, Tan KH, Li LJ. Application and utility of continuous glucose monitoring in pregnancy: a systematic review. Front Endocrinol (Lausanne). 2019;10:697. 